Article

\title{
Neutrosophic Multigroups and Applications
}

\author{
Vakkas Uluçay *(i) and Memet Şahin \\ Department of Mathematics, Gaziantep University, 27310 Gaziantep, Turkey; mesahin@gantep.edu.tr \\ * Correspondence: vulucay27@gmail.com; Tel.: +90-537-643-5034
}

Received: 12 December 2018; Accepted: 14 January 2019; Published: 17 January 2019

\begin{abstract}
In recent years, fuzzy multisets and neutrosophic sets have become a subject of great interest for researchers and have been widely applied to algebraic structures include groups, rings, fields and lattices. Neutrosophic multiset is a generalization of multisets and neutrosophic sets. In this paper, we proposed a algebraic structure on neutrosophic multisets is called neutrosophic multigroups which allow the truth-membership, indeterminacy-membership and falsity-membership sequence have a set of real values between zero and one. This new notation of group as a bridge among neutrosophic multiset theory, set theory and group theory and also shows the effect of neutrosophic multisets on a group structure. We finally derive the basic properties of neutrosophic multigroups and give its applications to group theory.
\end{abstract}

Keywords: neutrosophic sets; neutrosophic multisets; neutrosophic multigroups; neutrosophic multisubgroups

\section{Introduction}

In the real world, there are much uncertainty information which cannot be handled by crisp values. The fuzzy set theory [1] has been an age old and effective tool to tackle uncertainty information by introduced Zadeh but it can be applied only on random process. Therefore, on the basis of fuzzy set theory, Sebastian and Ramakrishnan [2] introduced Multi-Fuzzy Sets, Atanassov [3] proposed intuitionistic fuzzy set theory, Shinoj and John [4] initiated intuitionistic fuzzy multisets. Recently, the above theories have developed in many directions and found its applications in a wide variety of fields including algebraic structures. For example, on fuzzy sets [5-7], on fuzzy multi sets [8-10], on intuitionistic fuzzy sets [11-19], on intuitionistic fuzzy multi sets [20] are some of the selected works.

But these theories cannot manage the all types of uncertainties, such as indeterminate and inconsistent information some decision-making problems. For instance, "when we ask the opinion of an expert about certain statement, he or she may that the possibility that the statement is true is 0.5 and the statement is false is 0.6 and the degree that he or she is not sure is 0.2 " [21]. In order to overcome this shortage, Smarandache [22] introduced neutrosophic set theory to makes the theory of Atanassov [3] very convenient and easily applicable in practice. Then, Wang et al. [21] gave the some operations and results of single valued neutrosophic set theory. In order to establish the algebraic structures of neutrosophic sets, some authors gave definition of neutrosophic groups [23-26] that is actually a example of a group. To develop the neutrosophic set theory, the concept of neutrosophic multi sets was initiated by Deli et al. [27] and Ye [28,29] for modeling vagueness and uncertainty. Using their definitions, in this paper, we define a new type of neutrosophic group on a neutrosophic multi set, which we call neutrosophic multi set group. Since this new concept a brings the neutrosophic multi set theory, set theory and the group theory together, it is very functional in the sense of improving the neutrosophic multi set theory with respect to group structure. Rosenfeld [30] extended the classical group theory to fuzzy set. By using the definitions and results on fuzzy 
sets in [6,30] and on intuitionistic fuzzy multiset in [20], we applied the definitions and results to neutrosophic multi set theory.The above set theories have been applied to many different areas including neutrosophic environments have been studied by many researchers in [31-39]. In this paper the notion of neutrosophic multigroup along with some related properties have been introduced by follow the results of intuitionistic fuzzy group theory. This concept will bring a new opportunity in research and development of neutrosophic sets theory.

The paper is organized as follows. In Section 2, we briefly review some preliminary concepts that will be used in the paper. In Section 3, we introduce the concept of neutrosophic multi group and give several basic properties and operations. In Section 4, we give some applications to the group theory with respect to neutrosophic multi groups. In Section 5, we make some concluding remarks and suggest.

\section{Preliminary}

In this section, we present basic definitions of fuzzy set theory, multi fuzzy set theory, intuitionistic fuzzy set theory, intuitionistic fuzzy multi set theory, neutrosophic set theory and neutrosophic multi set theory. For more detailed explanations related to this section, we refer to the earlier studies $[1,2,4,6,20,22,27,30]$.

Definition 1 ([1]). Let E be a universe.

Then, a fuzzy set $X$ over $E$ is defined by

$$
X=\left\{\left(\mu_{X}(x) / x\right): x \in E\right\},
$$

where $\mu_{X}$ is called membership function of $X$ and defined by $\mu_{X}: E \rightarrow[0,1]$. For each $x \in E$, the value $\mu_{X}(x)$ represents the degree of $x$ belonging to the fuzzy set $X$.

Definition 2 ([2]). Let X be a non-empty set. A multi-fuzzy set A on X is defined as:

$$
A=\left\{<x, \mu_{1}(x), \mu_{2}(x), \mu_{3}(x), \ldots, \mu_{i} \ldots: x \in E\right\},
$$

where $\mu_{i}: X \rightarrow[0,1]$ for all $i \in\{1,2, \ldots, p\}$ and $x \in E$.

Definition 3 ([4]). Let X be a nonempty set. An Intuitionistic Fuzzy Multi-set A denoted by IFMS drawn from $X$ is characterized by two functions: 'count membership' of $A\left(C M_{A}\right)$ and 'count non membership' of $A\left(C N_{A}\right)$ given respectively by $A\left(C M_{A}\right): X \rightarrow Q$ and $A\left(C N_{A}\right): X \rightarrow Q$ where $Q$ is the set of all crisp multi-sets drawn from the unit interval $[0,1]$ such that, for each $x \in X$, the membership sequence is defined as a decreasingly ordered sequence of elements in $C M_{A}(x)$, which is denoted by $\left(\mu_{A}^{1}(x), \mu_{A}^{2}(x), \ldots, \mu_{A}^{P}(x)\right)$ where $\mu_{A}^{1}(x) \geq \mu_{A}^{2}(x) \geq \ldots \geq \mu_{A}^{P}(x)$ and the corresponding non membership sequence will be denoted by $\left(v_{A}^{1}(x), v_{A}^{2}(x), \ldots, v_{A}^{P}(x)\right)$ such that $0 \leq \mu_{A}^{i}(x)+v_{A}^{i}(x) \leq 1$ for every $x \in X$ and $i=(1,2,3, \ldots, p)$. An IFMS $A$ is denoted by

$$
A=\left\{\left\langle x:\left(\mu_{A}^{1}(x), \mu_{A}^{2}(x), \ldots, \mu_{A}^{P}(x)\right),\left(v_{A}^{1}(x), v_{A}^{2}(x), \ldots, v_{A}^{P}(x)\right)\right\rangle: x \in X\right\} .
$$

Definition 4 ([4]). Length of an element $x$ in an IFMS. A defined as the Cardinality of $C M_{A}(x)$ or $C N_{A}(x)$ for which $0 \leq \mu_{A}^{j}(x)+v_{A}^{j}(x) \leq 1$ and it is denoted by $L(x: A)$. That is,

$$
L(x: A)=\left|C M_{A}(x)\right|=\left|C N_{A}(x)\right| .
$$

Proposition 1 ([20]). Let $A, B, A_{i} \in \operatorname{IFMS}(X)$; then, the following results hold:

1. $\left[A^{-1}\right]^{-1}=A$.

2. $A \subseteq B \Rightarrow A^{-1} \subseteq B^{-1}$. 
3. $\left[\bigcup_{i=1}^{n} A_{i}\right]^{-1}=\bigcup_{i=1}^{n}\left[A_{i}^{-1}\right]$.

4. $\left[\bigcap_{i=1}^{n} A_{i}\right]^{-1}=\bigcap_{i=1}^{n}\left[A_{i}^{-1}\right]$.

5. $(A \circ B)^{-1}=B^{-1} \circ A^{-1}$.

6.

$$
\begin{aligned}
C M_{A \circ B}(x) & =\vee_{y \in X}\left\{C M_{A}(y) \wedge C M_{B}\left(y^{-1} x\right)\right\} \quad \forall x \in X \\
& =\vee_{y \in X}\left\{C M_{A}\left(x y^{-1}\right) \wedge C M_{B}(y)\right\} \forall x \in X . \\
C N_{A \circ B}(x) & =\wedge_{y \in X}\left\{C N_{A}(y) \vee C N_{B}\left(y^{-1} x\right)\right\} \forall x \in X \\
& =\wedge_{y \in X}\left\{C N_{A}\left(x y^{-1}\right) \vee C N_{B}(y)\right\} \forall x \in X .
\end{aligned}
$$

Definition 5 ([20]). Let X be a group. An intuitionistic fuzzy multiset $G$ over $X$ is an intuitionistic fuzzy multi group (IFMG) over $X$ if the counts(count membership and non membership) of $G$ satisfies the following four conditions:

1. $C M_{G}(x y) \geq C M_{G}(x) \wedge C M_{G}(y) \forall x, y \in X$.

2. $C M_{G}\left(x^{-1}\right) \geq C T_{G}(x) \forall x \in X$.

3. $C N_{G}(x y) \leq C N_{G}(x) \wedge C I_{G}(y) \forall x, y \in X$.

4. $C N_{G}\left(x^{-1}\right) \leq C N_{G}(x) \forall x \in X$.

Definition 6 ([22]). Let X be a space of points (objects), with a generic element in X denoted by $x$. A neutrosophic set $\left(N\right.$-set) $A$ in $X$ is characterized by a truth-membership function $T_{A}$, a indeterminacy-membership function $I_{A}$ and a falsity-membership function $F_{A} . T_{A}(x), I_{A}(x)$ and $F_{A}(x)$ are real standard or nonstandard subsets of $\left[{ }^{-} 0,1^{+}\right]$.

It can be written as

$$
A=\left\{<x,\left(T_{A}(x), I_{A}(x), F_{A}(x)\right)>: x \in X, T_{A}(x), I_{A}(x), F_{A}(x) \in[0,1]\right\} .
$$

There is no restriction on the sum of $T_{A}(x) ; I_{A}(x)$ and $F_{A}(x)$, so ${ }^{-} 0 \leq \sup _{A}(x)+\operatorname{supI}_{A}(x)+$ $\operatorname{supF}_{A}(x) \leq 3^{+}$.

Here, $1^{+}=1+\varepsilon$, where 1 is its standard part and $\varepsilon$ its non-standard part. Similarly, $-0=1+\varepsilon$, where 0 is its standard part and $\varepsilon$ its non-standard part.

Definition 7 ([27]). Let E be a universe. A neutrosophic multiset set(Nms) A on E can be defined as follows:

$$
\begin{array}{r}
A=\left\{<x,\left(T_{A}^{1}(x), T_{A}^{2}(x), \ldots, T_{A}^{P}(x)\right),\left(I_{A}^{1}(x), I_{A}^{2}(x), \ldots, I_{A}^{P}(x)\right),\right. \\
\left.\left(F_{A}^{1}(x), F_{A}^{2}(x), \ldots, F_{A}^{P}(x)\right)>: x \in E\right\},
\end{array}
$$

where

$$
\begin{gathered}
T_{A}^{1}(x), T_{A}^{2}(x), \ldots, T_{A}^{P}(x): E \rightarrow[0,1], \\
I_{A}^{1}(x), I_{A}^{2}(x), \ldots, I_{A}^{P}(x): E \rightarrow[0,1],
\end{gathered}
$$

and

$$
F_{A}^{1}(x), F_{A}^{2}(x), \ldots, F_{A}^{P}(x): E \rightarrow[0,1]
$$

such that

$$
0 \leq \operatorname{supT}_{A}^{i}(x)+\operatorname{supI}_{A}^{i}(x)+\operatorname{supF}_{A}^{i}(x) \leq 3
$$

$(i=1,2, \ldots, P)$ and

$$
T_{A}^{1}(x) \leq T_{A}^{2}(x) \leq \ldots \leq T_{A}^{P}(x)
$$

for any $x \in E$.

$\left(T_{A}^{1}(x), T_{A}^{2}(x), \ldots, T_{A}^{P}(x)\right), \quad\left(I_{A}^{1}(x), I_{A}^{2}(x), \ldots, I_{A}^{P}(x)\right) \quad$ and $\quad\left(F_{A}^{1}(x), F_{A}^{2}(x), \ldots, F_{A}^{P}(x)\right) \quad$ is the truth-membership sequence, indeterminacy-membership sequence and falsity-membership sequence of 
the element $x$, respectively. In addition, $P$ is called the dimension(cardinality) of Nms $A$, denoted $d(A)$. We arrange the truth-membership sequence in decreasing order, but the corresponding indeterminacy-membership and falsity-membership sequence may not be in decreasing or increasing order.

Definition $8([27,28])$. Let $A, B$ be two Nms. Then,

1. $A$ is said to be Nm-subset of $B$ is denoted by $A \subseteq \widetilde{\subseteq} B$ if $T_{A}^{i}(x) \leq T_{B}^{i}(x), I_{A}^{i}(x) \geq I_{B}^{i}(x), F_{A}^{i}(x) \geq F_{B}^{i}(x)$, $\forall x \in E$ and $i=1,2, \ldots, P$.

2. $A$ is said to be neutrosophic equal of $B$ is denoted by $A=B$ if $T_{A}^{i}(x)=T_{B}^{i}(x), I_{A}^{i}(x)=I_{B}^{i}(x)$, $F_{A}^{i}(x)=F_{B}^{i}(x), \forall x \in E$ and $i=1,2, \ldots, P$.

3. The union of $A$ and $B$ is denoted by $A \widetilde{\cup} B=C$ and is defined by

$$
C=\left\{<x,\left(T_{C}^{1}(x), T_{C}^{2}(x), \ldots, T_{C}^{P}(x)\right),\left(I_{C}^{1}(x), I_{C}^{2}(x), \ldots, I_{C}^{P}(x)\right),\left(F_{C}^{1}(x), F_{C}^{2}(x), \ldots, F_{C}^{P}(x)\right)>: x \in E\right\},
$$

where $T_{C}^{i}=T_{A}^{i}(x) \vee T_{B}^{i}(x), I_{C}^{i}=I_{A}^{i}(x) \wedge I_{B}^{i}(x), F_{C}^{i}=F_{A}^{i}(x) \wedge F_{B}^{i}(x), \forall x \in E$ and $i=1,2, \ldots, P$.

4. The intersection of $A$ and $B$ is denoted by $A \widetilde{\cap} B=D$ and is defined by

$$
D=\left\{<x,\left(T_{D}^{1}(x), T_{D}^{2}(x), \ldots, T_{D}^{P}(x)\right),\left(I_{D}^{1}(x), I_{D}^{2}(x), \ldots, I_{D}^{P}(x)\right),\left(F_{D}^{1}(x), F_{D}^{2}(x), \ldots, F_{D}^{P}(x)\right)>: x \in E\right\},
$$

where $T_{D}^{i}=T_{A}^{i}(x) \wedge T_{B}^{i}(x), I_{D}^{i}=I_{A}^{i}(x) \vee I_{B}^{i}(x), F_{D}^{i}=F_{A}^{i}(x) \vee F_{B}^{i}(x), \forall x \in E$ and $i=1,2, \ldots, P$.

\section{Neutrosophic Multigroups}

In this section, we introduce neutrosophic multigroups and investigate their basic properties. Throughout this section,

1. Let $X$ be a group with a binary operation and the identity element is $e$.

2. NMS $(X)$ denotes the set of all neutrosophic multisets over the $X$.

3. $N M G(X)$ denotes the set of all neutrosophic multi groups $N M G$ over the group $X$.

Definition 9. Let $X$ be a group $A \in N M S(X)$. Then, $A^{-1}$ is defined as

$$
\begin{array}{r}
A^{-1}=\left\{<x,\left(T_{A}^{1-1}(x), T_{A}^{2-1}(x), \ldots, T_{A}^{P}(x)\right)^{-1},\left(I_{A}^{1}(x)^{-1}, I_{A}^{2-1}(x), \ldots, I_{A}^{P^{-1}}(x)\right),\right. \\
\left.\left(F_{A}^{1-1}(x), F_{A}^{2-1}(x), \ldots, F_{A}^{P^{-1}}(x)\right)>: x \in E\right\},
\end{array}
$$

where $T_{A}^{i-1}(x)=T_{A}^{i}\left(x^{-1}\right), I_{A}^{i-1}(x)=I_{A}^{i}\left(x^{-1}\right)$ and $F_{A}^{i-1}(x)=F_{A}^{i}\left(x^{-1}\right)$ for all $i=1,2, \ldots, P$.

Definition 10. Let $X$ be a classical group $A \in N M S(X)$. Then, $A$ is called a neutrosophic multi groupoid over $X$ if

1. $T_{G}^{i}(x y) \geq T_{G}^{i}(x) \wedge T_{G}^{i}(y)$,

2. $I_{G}^{i}(x y) \leq I_{G}^{i}(x) \vee I_{G}^{i}(y)$,

3. $F_{G}^{i}(x y) \leq F_{G}^{i}(x) \vee F_{G}^{i}(y)$,

for all $x, y \in X$ and $i=1,2, \ldots, P$.

A is called a neutrosophic multi group(NM-group) over X if the neutrosophic multi groupoid satisfies

1. $T_{G}^{i}\left(x^{-1}\right) \geq T_{G}^{i}(x)$,

2. $I_{G}^{i}\left(x^{-1}\right) \leq I_{G}^{i}(x)$,

3. $F_{G}^{i}\left(x^{-1}\right) \leq F_{G}^{i}(x)$,

for all $x \in X$ and $i=1,2, \ldots, P$. 
Example 1. Assume that $\left(Z_{3},+\right)$ is a classical group. Then,

$$
\begin{aligned}
A= & \{\langle 0 ;(0.8,0.7,0.6,0.4),(0.1,0.1,0.2,0.3),(0.2,0.3,0.4,0.5)\rangle,\langle 1 ;(0.7,0.6,0.4,0.3), \\
& (0.2,0.3,0.2,0.3),(0.3,0.4,0.5,0.6)\rangle,\langle 2 ;(0.8,0.6,0.6,0.4),(0.1,0.2,0.2,0.3),(0.2,0.4,0.4,0.5)\rangle\}
\end{aligned}
$$

is a NM-group. However,

$$
\begin{aligned}
B= & \{\langle 0 ;(0.8,0.7,0.6,0.4),(0.1,0.1,0.2,0.3),(0.2,0.3,0.4,0.5)\rangle,\langle 1 ;(0.9,0.5,0.4,0.3),(0.2,0.1,0.2,0.3), \\
& (0.3,0.3,0.5,0.4)\rangle,\langle 2 ;(0.8,0.7,0.6,0.4),(0.1,0.3,0.2,0.3),(0.2,0.4,0.4,0.6)\rangle\}
\end{aligned}
$$

is not a NM-group because $T_{B}^{i}\left(1^{-1}\right)$ is not greater than or equal to $T^{i}{ }_{B}(1)$.

From the Definition 10 and Example 1, it is clear that a NM-group is a generalized case of fuzzy group and intuitionistic fuzzy multi group.

Proposition 2. Let $X$ be a classical group and $A \in N M S(X)$. If $A \in N M G(X)$; then,

1. $T_{A}^{i}(e) \geq T_{A}^{i}(x) \forall x \in X$,

2. $I^{i}{ }_{A}(e) \leq I^{i}{ }_{A}(x) \forall x \in X$,

3. $F^{i}{ }_{A}(e) \leq F^{i}{ }_{A}(x) \forall x \in X$,

for all $x \in X$ and $i=1,2, \ldots, P$.

Proof. Since A an NM - group over X, then

1.

$$
\begin{aligned}
T^{i}{ }_{A}(e) & =T^{i}{ }_{A}\left(x \cdot x^{-1}\right) \\
& \geq T^{i}{ }_{A}(x) \wedge T^{i}{ }_{A}\left(x^{-1}\right) \\
& \geq T^{i}{ }_{A}(x) \wedge T^{i}{ }_{A}(x) \\
& =T^{i}{ }_{A}(x)
\end{aligned}
$$

for all $x \in X$ and $i=1,2, \ldots, P$.

2.

$$
\begin{aligned}
I^{i}{ }_{A}(e) & =I^{i}{ }_{A}\left(x \cdot x^{-1}\right) \\
& \leq I^{i}{ }_{A}(x) \vee I^{i}{ }_{A}\left(x^{-1}\right) \\
& \leq I^{i}{ }_{A}(x) \vee I^{i}{ }_{A}(x) \\
& =I_{A}^{i}(x)
\end{aligned}
$$

for all $x \in X$ and $i=1,2, \ldots, P$.

3.

$$
\begin{aligned}
F^{i}{ }_{A}(e) & =F^{i}{ }_{A}\left(x . x^{-1}\right) \\
& \leq F^{i}{ }_{A}(x) \vee F^{i}{ }_{A}\left(x^{-1}\right) \\
& \leq F^{i}{ }_{A}(x) \vee F^{i}{ }_{A}(x) \\
& =F^{i}{ }_{A}(x)
\end{aligned}
$$

for all $x \in X$ and $i=1,2, \ldots, P$.

Proposition 3. Let $X$ be a classical group and $A \in N M S(X)$. If $A \in N M G(X)$, then

1. $T_{A}^{i}\left(x^{n}\right) \geq T_{A}^{i}(x) \forall x \in X$,

2. $I_{A}^{i}\left(x^{n}\right) \leq I^{i}{ }_{A}(x) \forall x \in X$,

3. $F_{A}^{i}\left(x^{n}\right) \leq F_{A}^{i}(x) \forall x \in X$,

for all $x \in X$ and $i=1,2, \ldots, P$. 
Proof. Since A an NM - group over X, then

1.

$$
\begin{aligned}
T^{i}{ }_{A}\left(x^{n}\right) & \geq T^{i}{ }_{A}(x) \wedge T^{i}{ }_{A}\left(x^{n-1}\right) \\
& \geq T^{i}{ }_{A}(x) \wedge T^{i}{ }_{A}(x) \wedge \ldots \wedge T_{A}^{i}(x) \\
& =T^{i}{ }_{A}(x)
\end{aligned}
$$

for all $x \in X$ and $i=1,2, \ldots, P$.

2.

$$
\begin{aligned}
I^{i}{ }_{A}\left(x^{n}\right) & \leq I^{i}{ }_{A}(x) \vee I^{i}{ }_{A}\left(x^{n-1}\right) \\
& \leq I^{i}{ }_{A}(x) \vee I^{i}{ }_{A}(x) \vee \ldots \vee I^{i}{ }_{A}(x) \\
& =I^{i}{ }_{A}(x)
\end{aligned}
$$

for all $x \in X$ and $i=1,2, \ldots, P$.

3.

$$
\begin{aligned}
F^{i}{ }_{A}\left(x^{n}\right) & \leq F^{i}{ }_{A}(x) \vee F^{i}{ }_{A}\left(x^{n-1}\right) \\
& \leq F^{i}{ }_{A}(x) \vee F^{i}{ }_{A}(x) \vee \ldots \vee F^{i}{ }_{A}(x) \\
& =F^{i}{ }_{A}(x)
\end{aligned}
$$

for all $x \in X$ and $i=1,2, \ldots, P$.

Definition 11. Let $Y$ be a subgroup of $X, B \in N M G(Y), B \subseteq A$ and $A \in N M G(X)$. If $B \in N M G(Y)$, then $B$ is called a neutrosophic multi subgroup of $A$ over $X$ and denoted by $B \tilde{\leq} A$.

Example 2. Assume that $\left(Z_{3},+\right)$ is a classical group. We define $A$ and $B$ neutrosophic multi group over $\left(Z_{3},+\right)$ by

$$
\begin{aligned}
A= & \{\langle 0 ;(0.4,0.3,0.3,0.2),(0.1,0.1,0.2,0.3),(0.2,0.3,0.4,0.6)\rangle, \\
& \langle 1 ;(0.6,0.5,0.3,0.2),(0.2,0.4,0.2,0.3),(0.3,0.2,0.5,0.6)\rangle, \\
& \langle 2 ;(0.8,0.7,0.5,0.4),(0.1,0.3,0.2,0.3),(0.2,0.1,0.4,0.5)\rangle\} . \\
B= & \{\langle 0 ;(0.4,0.3,0.3,0.2),(0.1,0.1,0.2,0.3),(0.2,0.3,0.4,0.6)\rangle, \\
& \langle 1 ;(0.6,0.5,0.3,0.2),(0.2,0.4,0.2,0.3),(0.3,0.2,0.5,0.6)\rangle\} .
\end{aligned}
$$

Then, $B$ is a neutrosophic multi subgroup of $A$ over $\left(Z_{3},+\right)$ and denoted by $B \tilde{\leq} A$.

Theorem 1. Let $X$ be a group $A \in N M S(X)$. Then, $A$ is an $N M-$ group if and only if $T^{i}{ }_{A}\left(x y^{-1}\right) \geq$ $T^{i}{ }_{A}(x) \wedge T^{i}{ }_{A}(y), I_{A}^{i}\left(x y^{-1}\right) \leq I^{i}{ }_{A}(x) \vee I^{i}{ }_{A}(y)$ and $F^{i}{ }_{A}\left(x y^{-1}\right) \leq F^{i}{ }_{A}(x) \vee F^{i}{ }_{A}(y)$ for all $x, y \in X$.

Proof. Assume that A is an NM - group over X. Then,

$$
\begin{aligned}
T^{i}{ }_{A}\left(x y^{-1}\right) & \geq T^{i}{ }_{A}(x) \wedge T^{i}{ }_{A}\left(y^{-1}\right) \\
& \geq T^{i}{ }_{A}(x) \wedge T_{A}^{i}(y)
\end{aligned}
$$

for all $x, y \in X$ and $i=1,2, \ldots, P$.

$$
\begin{aligned}
I^{i}{ }_{A}\left(x y^{-1}\right) & \leq I^{i}{ }_{A}(x) \vee I^{i}{ }_{A}\left(y^{-1}\right) \\
& \leq I^{i}{ }_{A}(x) \vee I^{i}{ }_{A}(y)
\end{aligned}
$$

for all $x, y \in X$ and $i=1,2, \ldots, P$.

$$
\begin{aligned}
F^{i}{ }_{A}\left(x y^{-1}\right) & \leq F^{i}{ }_{A}(x) \vee F^{i}{ }_{A}\left(y^{-1}\right) \\
& \leq F^{i}{ }_{A}(x) \vee F^{i}{ }_{A}(y)
\end{aligned}
$$

for all $x, y \in X$ and $i=1,2, \ldots, P$. 
Conversely, the given condition be satisfied. Firstly,

$$
\begin{aligned}
T_{A}^{i}\left(x^{-1}\right) & =T^{i}{ }_{A}\left(e . x^{-1}\right) \\
& \geq T^{i}{ }_{A}(e) \wedge T^{i}{ }_{A}(x) \\
& =T^{i}{ }_{A}(x) \\
T_{A}^{i}(x y) & \geq T^{i}{ }_{A}(x) \wedge T^{i}{ }_{A}\left(y^{-1}\right) \\
& \geq T_{A}^{i}(x) \wedge T^{i}(y) .
\end{aligned}
$$

Secondly,

$$
\begin{aligned}
I^{i}{ }_{A}\left(x^{-1}\right) & =I^{i}{ }_{A}\left(e \cdot x^{-1}\right) \\
& \leq I^{i}{ }_{A}(e) \vee I^{i}{ }_{A}(x) \\
& =I^{i}{ }_{A}(x) \\
I^{i}{ }_{A}(x y) & \leq I^{i}{ }_{A}(x) \vee I^{i}{ }_{A}\left(y^{-1}\right) \\
\leq & I^{i}{ }_{A}(x) \vee I^{i}{ }_{A}(y) .
\end{aligned}
$$

Thirdly,

$$
\begin{aligned}
F^{i}{ }_{A}\left(x^{-1}\right) & =F^{i}{ }_{A}\left(e \cdot x^{-1}\right) \\
& \leq F^{i}{ }_{A}(e) \vee F^{i}{ }_{A}(x) \\
& =F^{i}{ }_{A}(x) \\
F^{i}{ }_{A}(x y) \leq & F^{i}{ }_{A}(x) \vee F^{i}{ }_{A}\left(y^{-1}\right) \\
\leq & F^{i}{ }_{A}(x) \vee F^{i}{ }_{A}(y)
\end{aligned}
$$

so the proof is complete.

Definition 12. Let $A, B \in N M S(X)$. Then, their " $A N D$ " operation is denoted by $A \tilde{\wedge} B$ and is defined by

$$
A \tilde{\wedge} B=\left\{(x, y), T_{A \wedge B}^{i}(x, y), I_{A \wedge B}^{i}(x, y), F_{A \wedge B}^{i}(x, y):(x, y) \in X \times X\right\},
$$

where $T_{A \wedge B}^{i}(x, y)=T_{A}^{i}(x) \wedge T_{B}^{i}(y), I_{A \wedge B}^{i}(x, y)=I_{A}^{i}(x) \vee I_{B}^{i}(y), F_{A \wedge B}^{i}(x, y)=F_{A}^{i}(x) \vee F_{B}^{i}(y)$.

Theorem 2. Let $A, B \in N M G(X)$. Then, $A \tilde{\wedge} B$ is a neutrosophic multi group over $X$.

Proof. Let $\left(x_{1}, y_{1}\right),\left(x_{2}, y_{2}\right) \in X \times X$. Then,

$$
\begin{aligned}
T_{A \wedge B}^{i}\left(\left(x_{1}, y_{1}\right),\left(x_{2}, y_{2}\right)^{-1}\right) & =T^{i}{ }_{A{ }_{A} B}\left(x_{1} x_{2}^{-1}, y_{1} y_{2}^{-1}\right) \\
& =T^{i}{ }_{A}\left(x_{1} x_{2}^{-1}\right) \wedge T^{i}{ }_{B}\left(y_{1} y_{2}^{-1}\right) \\
& \geq\left(T^{i}{ }_{A}\left(x_{1}\right) \wedge T^{i}{ }_{A}\left(x_{2}\right)\right) \wedge\left(T^{i}{ }_{B}\left(y_{1}\right) \wedge T^{i}{ }_{B}\left(y_{2}\right)\right) \\
& =\left(T^{i}{ }_{A}\left(x_{1}\right) \wedge T^{i} i_{B}\left(y_{1}\right)\right) \wedge\left(T^{i}{ }_{A}\left(x_{2}\right) \wedge T^{i}{ }_{B}\left(y_{2}\right)\right) \\
& =T^{i}{ }_{A \wedge B}\left(x_{1}, y_{1}\right) \wedge T^{i}{ }_{A \wedge B}\left(x_{2}, y_{2}\right) \\
I_{A \wedge B}^{i}\left(\left(x_{1}, y_{1}\right),\left(x_{2}, y_{2}\right)^{-1}\right) & =I^{i}{ }_{A \wedge B}\left(x_{1} x_{2}^{-1}, y_{1} y_{2}^{-1}\right) \\
& =I^{i}{ }_{A}\left(x_{1} x_{2}^{-1}\right) \vee I_{B}^{i}{ }_{B}\left(y_{1} y_{2}^{-1}\right) \\
& \leq\left(I^{i}{ }_{A}\left(x_{1}\right) \vee I_{A}^{i}\left(x_{2}\right)\right) \vee\left(I_{B}^{i}\left(y_{1}\right) \vee I_{B}^{i}\left(y_{2}\right)\right) \\
& =\left(I^{i}{ }_{A}\left(x_{1}\right) \vee I^{i}{ }_{B}\left(y_{1}\right)\right) \vee\left(I^{i}{ }_{A}\left(x_{2}\right) \vee I^{i}{ }_{B}\left(y_{2}\right)\right) \\
& =I^{i}{ }_{A \wedge B}\left(x_{1}, y_{1}\right) \vee I^{i}{ }_{A \wedge B}\left(x_{2}, y_{2}\right)
\end{aligned}
$$

and

$$
\begin{aligned}
F_{A \wedge B}^{i}\left(\left(x_{1}, y_{1}\right),\left(x_{2}, y_{2}\right)^{-1}\right) & =F_{A \wedge B}^{i}\left(x_{1} x_{2}^{-1}, y_{1} y_{2}^{-1}\right) \\
& =F_{A}^{i}\left(x_{1} x_{2}^{-1}\right) \vee F^{i}{ }_{B}\left(y_{1} y_{2}^{-1}\right) \\
& \leq\left(F^{i}{ }_{A}\left(x_{1}\right) \vee F^{i}{ }_{A}\left(x_{2}\right)\right) \vee\left(F_{B}^{i}\left(y_{1}\right) \vee F_{B}^{i}\left(y_{2}\right)\right) \\
& =\left(F^{i}{ }_{A}\left(x_{1}\right) \vee F^{i}{ }_{B}\left(y_{1}\right)\right) \vee\left(F^{i}{ }_{A}\left(x_{2}\right) \vee F^{i}{ }_{B}\left(y_{2}\right)\right) \\
& =F_{A \wedge B}^{i}\left(x_{1}, y_{1}\right) \vee F_{A \wedge B}^{i}\left(x_{2}, y_{2}\right)
\end{aligned}
$$


for all $\left(x_{1}, y_{1}\right),\left(x_{2}, y_{2}\right) \in X$ and $i=1,2, \ldots, P$. Therefore, $A \tilde{\wedge} B$ is a neutrosophic multi group over $X$, hence the proof.

Example 3. Let us take into consideration the classical group $\left(Z_{3},+\right)$. Define the neutrosophic multiset $A, B$ on $\left(Z_{3},+\right)$ as follows:

$$
\begin{aligned}
& A=\{\langle 0 ;(0.5,0.3,0.2,0.1),(0.1,0.1,0.2,0.3),(0.2,0.3,0.4,0.5)\rangle, \\
&\langle 1 ;(0.6,0.4,0.3,0.2),(0.1,0.3,0.3,0.4),(0.1,0.2,0.4,0.6)\rangle, \\
&\langle 2 ;(0.7,0.5,0.3,0.2),(0.2,0.2,0.3,0.4),(0.3,0.3,0.4,0.6)\rangle, \\
& B=\{\langle 0 ;(0.6,0.5,0.4,0.2),(0.2,0.2,0.3,0.4),(0.3,0.3,0.4,0.6)\rangle, \\
&\langle 1 ;(0.8,0.6,0.4,0.3),(0.1,0.1,0.2,0.3),(0.2,0.2,0.3,0.4)\rangle\} \\
& \text { are NM - groups. } \\
& A \tilde{\wedge} B \quad=\{\langle(0,0) ;(0.5,0.3,0.2,0.1),(0.2,0.2,0.2,0.4),(0.2,0.3,0.4,0.6)\rangle, \\
&\langle(0,1) ;(0.5,0.3,0.2,0.1),(0.1,0.1,0.2,0.3),(0.2,0.2,0.3,0.5)\rangle, \\
&\langle(1,0) ;(0.6,0.4,0.3,0.2),(0.2,0.2,0.3,0.4),(0.3,0.3,0.4,0.6)\rangle, \\
&\langle(1,1) ;(0.6,0.4,0.3,0.2),(0.1,0.3,0.3,0.4),(0.2,0.2,0.4,0.6)\rangle, \\
&\langle(2,0) ;(0.6,0.5,0.3,0.2),(0.2,0.2,0.3,0.4),(0.3,0.3,0.4,0.6)\rangle, \\
&\langle(2,1) ;(0.7,0.5,0.3,0.2),(0.2,0.2,0.3,0.4),(0.3,0.3,0.4,0.6)\rangle\} .
\end{aligned}
$$

Then, $A \tilde{\wedge} B \in N M G(X)$.

Definition 13. Let $X$ be a classical group and $A, B \in N M S(X)$. Then, their "OR" operation is denoted by $A \tilde{\nabla} B$ and is defined by

$$
A \tilde{\nabla} B=\left\{(x, y), T_{A \sim B}^{i}(x, y), I_{A \vee B}^{i}(x, y), F_{A \tilde{}}^{i} B(x, y):(x, y) \in X \times X\right\}
$$

where $T^{i}{ }_{A \tilde{\vee} B}(x, y)=T^{i}{ }_{A}(x) \vee T^{i}{ }_{B}(y), I_{A \tilde{}}^{i} B(x, y)=I_{A}^{i}(x) \wedge I_{B}^{i}(y), F_{A \tilde{}}^{i}(x, y)=F_{A}^{i}(x) \wedge F_{B}^{i}(y)$.

Proposition 4. Let $A, B \in N M G(X)$. Then, $T_{A \vee B}^{i}(x) \leq T_{A \vee B}^{i}\left(x^{-1}\right), I_{A \vee B}^{i}(x) \geq I_{A \vee B}^{i}\left(x^{-1}\right)$, $F_{A \approx B}^{i}(x) \geq F_{A \vee B}^{i}\left(x^{-1}\right)$.

Proof. Let $\left(x_{1}, y_{1}\right),\left(x_{2}, y_{2}\right) \in X \times X$. Then,

$$
\begin{aligned}
& T_{A \tilde{}}^{i} B\left(\left(x_{1}, y_{1}\right),\left(x_{2}, y_{2}\right)^{-1}\right)=T_{A \tilde{\vee} B}^{i}\left(x_{1} x_{2}^{-1}, y_{1} y_{2}^{-1}\right) \\
& =T_{A}^{i}\left(x_{1} x_{2}^{-1}\right) \vee T_{B}^{i}\left(y_{1} y_{2}^{-1}\right) \\
& \leq\left(T^{i}{ }_{A}\left(x_{1}\right) \vee T^{i}{ }_{A}\left(x_{2}\right)\right) \vee\left(T^{i}{ }_{B}\left(y_{1}\right) \vee T^{i}{ }_{B}\left(y_{2}\right)\right) \\
& =\left(T^{i}{ }_{A}\left(x_{1}\right) \vee T_{B}^{i}\left(y_{1}\right)\right) \vee\left(T^{i}{ }_{A}\left(x_{2}\right) \vee T^{i}{ }_{B}\left(y_{2}\right)\right) \\
& =T_{A \vee B}^{i}\left(x_{1}, y_{1}\right) \vee T_{A \vee B}^{i}\left(x_{2}, y_{2}\right) \\
& I_{A \tilde{}}^{i} B\left(\left(x_{1}, y_{1}\right),\left(x_{2}, y_{2}\right)^{-1}\right)=I_{A \tilde{}}^{i} B\left(x_{1} x_{2}^{-1}, y_{1} y_{2}^{-1}\right) \\
& =I^{i}{ }_{A}\left(x_{1} x_{2}^{-1}\right) \wedge I_{B}^{i}\left(y_{1} y_{2}^{-1}\right) \\
& \geq\left(I^{i}{ }_{A}\left(x_{1}\right) \wedge I^{i}{ }_{A}\left(x_{2}\right)\right) \wedge\left(I_{B}^{i}\left(y_{1}\right) \wedge I_{B}^{i}\left(y_{2}\right)\right) \\
& =\left(I^{i}{ }_{A}\left(x_{1}\right) \wedge I_{B}^{i}\left(y_{1}\right)\right) \wedge\left(I^{i}{ }_{A}\left(x_{2}\right) \wedge I_{B}{ }_{B}\left(y_{2}\right)\right) \\
& =I_{A \tilde{}}^{i}\left(x_{1}, y_{1}\right) \wedge I_{A \tilde{}}^{i} B\left(x_{2}, y_{2}\right)
\end{aligned}
$$

and

$$
\begin{aligned}
F_{A \tilde{\vee} B}^{i}\left(\left(x_{1}, y_{1}\right),\left(x_{2}, y_{2}\right)^{-1}\right) & =F^{i}{ }_{A \tilde{}}\left(x_{1} x_{2}^{-1}, y_{1} y_{2}^{-1}\right) \\
& =F^{i}{ }_{A}\left(x_{1} x_{2}^{-1}\right) \wedge F^{i}{ }_{B}\left(y_{1} y_{2}^{-1}\right) \\
& \geq\left(F^{i}{ }_{A}\left(x_{1}\right) \wedge F^{i}{ }_{A}\left(x_{2}\right)\right) \wedge\left(F_{B}^{i}\left(y_{1}\right) \wedge F_{B}^{i}\left(y_{2}\right)\right) \\
& =\left(F^{i}{ }_{A}\left(x_{1}\right) \wedge F_{B}^{i}\left(y_{1}\right)\right) \wedge\left(F^{i}{ }_{A}\left(x_{2}\right) \wedge F_{B}^{i}\left(y_{2}\right)\right) \\
& =F^{i}{ }_{A \tilde{}}\left(x_{1}, y_{1}\right) \wedge F_{A \tilde{}}^{i}\left(x_{2}, y_{2}\right)
\end{aligned}
$$


for all $\left(x_{1}, y_{1}\right),\left(x_{2}, y_{2}\right) \in X$ and $i=1,2, \ldots, P$-hence the proof.

From this, it is clear that, if $A, B \in N M G(X)$, then $A \tilde{\vee} B \in N M G(X)$ iff $T_{A \tilde{\vee} B}^{i}(x, y) \geq T_{A \tilde{}}^{i} B(x) \wedge$ $T_{A \tilde{\vee} B}^{i}(y), I_{A \tilde{}}^{i} B(x, y) \leq I_{A \tilde{}}^{i} B(x) \vee I_{A \tilde{}}^{i} B(y), F_{A \tilde{\vee} B}^{i}(x, y) \leq F_{A \tilde{}}^{i} B(x) \vee F_{A \tilde{}}^{i}(y)$.

Corollary 1. Let $A, B \in N M G(X)$. Then, $A \tilde{\nabla} B$ need not be an element of $N M G(X)$.

Example 4. Let us take into consideration the classical group $\left(Z_{4},+\right)$. Define the neutrosophic multiset $A, B$ on $\left(Z_{4},+\right)$ as follows:

$$
\begin{aligned}
& A=\{\langle 0 ;(0.5,0.3,0.2,0.1),(0.1,0.1,0.2,0.3),(0.2,0.3,0.4,0.5)\rangle, \\
&\langle 1 ;(0.6,0.4,0.3,0.2),(0.1,0.3,0.3,0.4),(0.1,0.2,0.4,0.6)\rangle, \\
&\langle 2 ;(0.7,0.5,0.3,0.2),(0.2,0.2,0.3,0.4),(0.3,0.3,0.4,0.6)\rangle, \\
&\langle 3 ;(0.7,0.6,0.4,0.3),(0.2,0.1,0.2,0.3),(0.3,0.2,0.1,0.3)\rangle\} \\
& B=\{\langle 0 ;(0.6,0.5,0.4,0.2),(0.2,0.2,0.3,0.4),(0.2,0.3,0.4,0.6)\rangle, \\
&\langle 1 ;(0.8,0.6,0.4,0.3),(0.1,0.1,0.2,0.3),(0.2,0.2,0.3,0.4)\rangle\} \\
& \text { are NM - groups. } \\
& A \tilde{\nabla} B \quad=\{\langle(0,0) ;(0.6,0.5,0.4,0.2),(0.1,0.1,0.2,0.3),(0.2,0.3,0.4,0.5)\rangle, \\
&\langle(0,1) ;(0.8,0.6,0.4,0.3),(0.1,0.1,0.2,0.3),(0.2,0.2,0.3,0.4)\rangle, \\
&\langle(1,0) ;(0.6,0.5,0.4,0.2),(0.1,0.2,0.3,0.4),(0.1,0.2,0.4,0.6)\rangle, \\
&\langle(1,1) ;(0.8,0.6,0.4,0.3),(0.1,0.1,0.2,0.3),(0.1,0.2,0.3,0.4)\rangle, \\
&\langle(2,0) ;(0.7,0.5,0.4,0.2),(0.1,0.2,0.3,0.4),(0.2,0.3,0.4,0.6)\rangle, \\
&\langle(2,1) ;(0.8,0.6,0.4,0.3),(0.1,0.1,0.2,0.3),(0.2,0.2,0.3,0.4)\rangle, \\
&\langle(3,0) ;(0.7,0.6,0.4,0.3),(0.2,0.1,0.2,0.3),(0.2,0.2,0.1,0.3)\rangle, \\
&\langle(3,1) ;(0.8,0.6,0.4,0.3),(0.1,0.1,0.2,0.3),(0.2,0.2,0.1,0.3)\rangle\} .
\end{aligned}
$$

However, $T_{A \tilde{}}^{i} B(3,0) \geq T_{A \tilde{}}^{i} B(1,0)$. Then, $A \tilde{\nabla} B \notin N M G(X)$.

Theorem 3. Let $X$ be a classical group and $A \in N M G(X)$. Then, the followings are equivalent:

1. $T_{A}^{i}(y x)=T_{A}^{i}(x y), I_{A}^{i}(y x)=I_{A}^{i}(x y)$ and $F_{A}^{i}(y x)=F_{A}^{i}(x y)$ for all $x, y \in X$.

2. $T_{A}^{i}\left(x y x^{-1}\right)=T_{A}^{i}(y), I_{A}^{i}\left(x y x^{-1}\right)=I_{A}^{i}(y)$ and $F_{A}^{i}\left(x y x^{-1}\right)=F_{A}^{i}(y)$ for all $x, y \in X$.

3. $\quad T_{A}^{i}\left(x y x^{-1}\right) \geq T_{A}^{i}(y), I_{A}^{i}\left(x y x^{-1}\right) \leq I_{A}^{i}(y)$ and $F_{A}^{i}\left(x y x^{-1}\right) \leq F_{A}^{i}(y)$ for all $x, y \in X$.

4. $T_{A}^{i}\left(x y x^{-1}\right) \leq T_{A}^{i}(y), I_{A}^{i}\left(x y x^{-1}\right) \geq I_{A}^{i}(y)$ and $F_{A}^{i}\left(x y x^{-1}\right) \geq F_{A}^{i}(y)$ for all $x, y \in X$.

Proof. 1. $\quad(1) \Rightarrow(2)$ : Let $x, y \in X$. Then,

$$
\begin{aligned}
T_{A}^{i}\left(x y x^{-1}\right) & =T_{A}^{i}\left(x^{-1} x y\right)=T_{A}^{i}(y), \\
I_{A}^{i}\left(x y x^{-1}\right) & =I_{A}^{i}\left(x^{-1} x y\right)=I_{A}^{i}(y), \\
F_{A}^{i}\left(x y x^{-1}\right) & =F_{A}^{i}\left(x^{-1} x y\right)=F_{A}^{i}(y) .
\end{aligned}
$$

2. $\quad(2) \Rightarrow(3)$ : Immediate.

3. $(3) \Rightarrow(4)$

$$
\begin{aligned}
& T_{A}^{i}\left(x y x^{-1}\right) \leq T_{A}^{i}\left(x^{-1} x y\left(x^{-1}\right)^{-1}\right)=T_{A}^{i}(y), \\
& I_{A}^{i}\left(x y x^{-1}\right) \geq I_{A}^{i}\left(x^{-1} x y\left(x^{-1}\right)^{-1}\right)=I_{A}^{i}(y), \\
& F_{A}^{i}\left(x y x^{-1}\right) \geq F_{A}^{i}\left(x^{-1} x y\left(x^{-1}\right)^{-1}\right)=F_{A}^{i}(y) .
\end{aligned}
$$

4. $(4) \Rightarrow(1)$ : Let $x, y \in X$. Then,

$$
\begin{aligned}
T_{A}^{i}(x y)= & T_{A}^{i}\left(x \cdot y x \cdot x^{-1}\right) \\
& \leq T_{A}^{i}(y x) \\
= & T_{A}^{i}\left(y \cdot x y \cdot y^{-1}\right) \\
& \leq T_{A}^{i}(x y)
\end{aligned}
$$




$$
\begin{aligned}
I_{A}^{i}(x y)= & I_{A}^{i}\left(x \cdot y x \cdot x^{-1}\right) \\
& \leq I_{A}^{i}(y x) \\
= & I_{A}^{i}\left(y \cdot x y \cdot y^{-1}\right) \\
& \leq I_{A}^{i}(x y), \\
F_{A}^{i}(x y)= & F_{A}^{i}\left(x \cdot y x \cdot x^{-1}\right) \\
& \leq F_{A}^{i}(y x) \\
= & F_{A}^{i}\left(y \cdot x y \cdot y^{-1}\right) \\
& \leq F_{A}^{i}(x y)
\end{aligned}
$$

Hence, $T_{A}^{i}(y x)=T_{A}^{i}(x y), I_{A}^{i}(y x)=I_{A}^{i}(x y), F_{A}^{i}(y x)=F_{A}^{i}(x y)$.

Definition 14. Let $X$ be a group, $A \in N M S(X)$ and $B$ is a nonempty neutrosophic multi subset of $A$ over $X$. Then, $B$ is called an abelian neutrosophic multi subset of $A$ if $T_{A}^{i}(y x)=T_{A}^{i}(x y), I_{A}^{i}(y x)=$ $I_{A}^{i}(x y)$ and $F_{A}^{i}(y x)=F_{A}^{i}(x y)$ for all $x, y \in X$.

Example 5. $1_{X}$ and $1_{e}$ are normal neutrosophic multi subgroup of $X$. If $X$ is a commutative group, every neutrosophic multi subgroup of $X$ is normal.

Definition 15. Let $X$ be a group, $A \in N M G(X)$ and $B$ is a neutrosophic multi subgroup of $A$ over $X$. Then, $B$ is called an a normal neutrosophic multi subgroup of $A$, denoted by $B \tilde{\nabla} A$ if it is an abelian neutrosophic multi subset of A over X.

Example 6. Assume that $\left(Z_{3},+\right)$ is a classiccal group. Define the neutrosophic multisets $A$ and $B$ on $\left(Z_{3},+\right)$ as follows:

$$
\begin{aligned}
A \quad & =\{\langle 0 ;(0.6,0.5,0.4,0.2),(0.1,0.1,0.2,0.3),(0.2,0.3,0.4,0.6)\rangle, \\
& \langle 1 ;(0.5,0.4,0.4,0.3),(0.2,0.1,0.2,0.3),(0.3,0.4,0.5,0.6)\rangle, \\
& \langle 2 ;(0.9,0.7,0.6,0.5),(0.1,0.1,0.2,0.3),(0.2,0.2,0.3,0.5)\rangle\}
\end{aligned}
$$

is a NM-group. If

$$
\begin{aligned}
B= & =\{\langle 0 ;(0.6,0.5,0.4,0.2),(0.1,0.1,0.2,0.3),(0.2,0.3,0.4,0.6)\rangle, \\
& \langle 1 ;(0.5,0.4,0.4,0.3),(0.2,0.1,0.2,0.3),(0.3,0.3,0.5,0.4)\rangle\},
\end{aligned}
$$

then $B$ is a neutrosophic multi subgroup of $A$ over $\left(Z_{3},+\right)$ and denoted by $B \tilde{\leq} A$. Therefore, $B \tilde{\nabla} A$.

Corollary 2. Let $A \in N M G(X)$ and $B$ be a neutrosophic multi subgroup of $A$ over $X$. If $X$ is an abelian group, then $B$ is a normal neutrosophic multi subgroup of $A$ over $X$.

\section{Applications of Neutrosophic Multi Groups}

In this section, we give some applications to the group theory with respect to neutrosophic multi groups.

Definition 16. Let $A$ be a neutrosophic multiset on $X$ and $\alpha \in[0,1]$. Define the $\alpha$-level sets of $A$ as follows:

$$
\begin{aligned}
& \left(T^{i}{ }_{A}\right)_{\alpha}=\left\{x \in X: T^{i}{ }_{A}(x) \geq \alpha\right\}, \\
& \left(I_{A}^{i}\right)^{\alpha}=\left\{x \in X: I_{A}^{i}(x) \leq \alpha\right\}, \\
& \left(F^{i}{ }_{A}\right)^{\alpha}=\left\{x \in X: F^{i} A(x) \leq \alpha\right\} .
\end{aligned}
$$


It is easy to verify that

(1) If $A \subseteq \tilde{\simeq} B$ and $\alpha \in[0,1]$, then $\left(T^{i}{ }_{A}\right)_{\alpha} \subseteq\left(T_{B}^{i}\right)_{\alpha},\left(I_{A}^{i}\right)^{\alpha} \supseteq\left(I_{B}^{i}\right)^{\alpha}$ and $\left(F_{A}^{i}\right)^{\alpha} \supseteq\left(F_{B}^{i}\right)^{\alpha}$.

(2) $\alpha \leq \beta$ implies $\left(T_{A}^{i}\right)_{\alpha} \supseteq\left(T_{A}^{i}\right)_{\beta},\left(I_{A}^{i}\right)^{\alpha} \subseteq\left(I_{A}^{i}\right)^{\beta}$ and $\left(F_{A}^{i}\right)^{\alpha} \subseteq\left(F_{A}^{i}\right)^{\beta}$.

Proposition 5. $A$ is a neutrosophic multi group of a classical group $X$ if and only if for all $\alpha \in[0,1], \alpha$-level sets of $A,\left(T_{A}^{i}\right)_{\alpha},\left(I_{A}^{i}\right)_{\alpha}$ and $\left(F_{A}^{i}\right)^{\alpha}$ are classical subgroups of $X$.

Proof. Let $A$ be a neutrosophic multi subgroup of $X, \alpha \in[0,1]$ and $x . y \in\left(T_{A}^{i}\right)_{\alpha}($ similarly $\left.x . y \in\left(I_{A}^{i}\right)_{\alpha},\left(F_{A}^{i}\right)^{\alpha}\right)$. By the assumption, $T_{A}^{i}\left(x y^{-1}\right) \geq T_{A}^{i}(x) \wedge T_{A}^{i}(y) \geq \alpha \wedge \alpha=\alpha$ (and similarly, $I_{A}^{i}\left(x y^{-1}\right) \leq \alpha$ and $\left.F_{A}^{i}\left(x y^{-1}\right) \leq \alpha\right)$. Hence, $x y^{-1} \in\left(T_{A}^{i}\right)_{\alpha}$ (and similarly $\left.x y^{-1} \in\left(I_{A}^{i}\right)^{\alpha},\left(F_{A}^{i}\right)^{\alpha}\right)$ for each $\alpha \in[0,1]$. This means that $\left(T_{A}^{i}\right)_{\alpha}$ (and similarly $\left.\left(I_{A}^{i}\right)^{\alpha},\left(F_{A}^{i}\right)^{\alpha}\right)$ is a classical subgroup of $X$ for each $\alpha \in[0,1]$.

Conversely, let $\left(T_{A}^{i}\right)_{\alpha}$ be a classical subgroup of $X$, for each $\alpha \in[0,1]$. Let $x, y \in X, \alpha=T_{A}^{i}(x) \wedge$ $T_{A}^{i}(y)$ and $\beta=T_{A}^{i}(x)$. Since $\left(T_{A}^{i}\right)_{\alpha}$ and $\left(T_{A}^{i}\right)_{\beta}$ are classical subgroups of $X, x . y \in\left(T_{A}^{i}\right)_{\alpha}$ and $x^{-1} \in\left(T_{A}^{i}\right)_{\beta}$. Thus, $T_{A}^{i}\left(x y^{-1}\right) \geq \alpha=T_{A}^{i}(x) \wedge T_{A}^{i}(y)$ and $T_{A}^{i}\left(x^{-1}\right) \geq \beta=T_{A}^{i}(x)$. Similarly, $I_{A}^{i}\left(x y^{-1}\right) \leq I_{A}^{i}(x) \vee I_{A}^{i}(y)$ and $F_{A}^{i}\left(x y^{-1}\right) \leq F_{A}^{i}(x) \vee F_{A}^{i}(y)$.

Theorem 4. Let $X_{1}, X_{2}$ be the classical groups and $g: X_{1} \rightarrow X_{2}$ be a group homomorphism. If $A$ is a neutrosophic multi subgroup of $X_{1}$, then the image of $A, g(A)$ is a neutrosophic multi subgroup of $X_{2}$.

Proof. Let $A \in N M S\left(X_{1}\right)$ and $y_{1}, y_{2} \in X_{2}$. If $g^{-1}\left(y_{1}\right)=\varnothing$ or $g^{-1}\left(y_{2}\right)=\varnothing$, then it is clear that $g(A) \in N M S\left(X_{2}\right)$. Let us assume that there exists $x_{1}, x_{2} \in X_{1}$ such that $g\left(x_{1}\right)=y_{1}$ and $g\left(x_{2}\right)=y_{2}$. Since $g$ is a group homomorphism,

$$
\begin{aligned}
& g\left(T_{A}^{i}\right)\left(y_{1} y_{2}^{-1}\right)=\bigvee_{y_{1} y_{2}^{-1}=g(x)} T_{A}^{i}(x) \geq T_{A}^{i}\left(x_{1} x_{2}^{-1}\right), \\
& g\left(I_{A}^{i}\right)\left(y_{1} y_{2}^{-1}\right)=\bigwedge_{y_{1} y_{2}^{-1}=g(x)} I_{A}^{i}(x) \leq I_{A}^{i}\left(x_{1} x_{2}^{-1}\right), \\
& g\left(F_{A}^{i}\right)\left(y_{1} y_{2}^{-1}\right)=\bigwedge_{y_{1} y_{2}^{-1}=g(x)} F_{A}^{i}(x) \leq F_{A}^{i}\left(x_{1} x_{2}^{-1}\right) .
\end{aligned}
$$

By using the above inequalities, let us prove that $g(A)\left(y_{1} y_{2}^{-1}\right) \geq g(A)\left(y_{1}\right) \wedge g(A)\left(y_{2}\right)$ :

$$
\begin{aligned}
& g(A)\left(y_{1} y_{2}^{-1}\right)=g\left(T_{A}^{i}\right)\left(y_{1} y_{2}^{-1}\right), g\left(I_{A}^{i}\right)\left(y_{1} y_{2}^{-1}\right), g\left(F_{A}^{i}\right)\left(y_{1} y_{2}^{-1}\right) \\
& =\bigvee_{y_{1} y_{2}^{-1}=g(x)} T_{A}^{i}(x), \bigwedge_{y_{1} y_{2}^{-1}=g(x)} I_{A}^{i}(x), \bigwedge_{y_{1} y_{2}^{-1}=g(x)} F_{A}^{i}(x) \\
& \geq\left(T_{A}^{i}\left(x_{1} x_{2}^{-1}\right), I_{A}^{i}\left(x_{1} x_{2}^{-1}\right), F_{A}^{i}\left(x_{1} x_{2}^{-1}\right)\right) \\
& \geq\left(T_{A}^{i}\left(x_{1}\right) \wedge T_{A}^{i}\left(x_{2}\right), I_{A}^{i}\left(x_{1}\right) \vee I_{A}^{i}\left(x_{2}\right), F_{A}^{i}\left(x_{1}\right) \vee F_{A}^{i}\left(x_{2}\right)\right. \\
& =\left(T_{A}^{i}\left(x_{1}\right), I_{A}^{i}\left(x_{1}\right), F_{A}^{i}\left(x_{1}\right)\right) \wedge\left(T_{A}^{i}\left(x_{2}\right), I_{A}^{i}\left(x_{2}\right), F_{A}^{i}\left(x_{2}\right)\right) \text {. }
\end{aligned}
$$

This is satisfied for each $x_{1}, x_{2} \in X_{1}$ with $g\left(x_{1}\right)=y_{1}$ and $g\left(x_{2}\right)=y_{2}$, then it is obvious that

$$
\begin{aligned}
& g(A)\left(y_{1} y_{2}^{-1}\right) \geq\left(\bigvee_{y_{1}=g\left(x_{1}\right)} T_{A}^{i}\left(x_{1}\right), \bigwedge_{y_{1}=g\left(x_{1}\right)} I_{A}^{i}\left(x_{1}\right), \bigwedge_{y_{1}=g\left(x_{1}\right)} F_{A}^{i}\left(x_{1}\right)\right) \\
& \wedge\left(\bigvee_{y_{2}=g\left(x_{2}\right)} T_{A}^{i}\left(x_{2}\right), \bigwedge_{y_{2}=g\left(x_{2}\right)} I_{A}^{i}\left(x_{2}\right), \bigwedge_{y_{2}}=g\left(x_{2}\right) F_{A}^{i}\left(x_{2}\right)\right) \\
& =\left(g\left(T_{A}^{i}\right)\left(y_{1}\right), g\left(I_{A}^{i}\right)\left(y_{1}\right), g\left(F_{A}^{i}\right)\left(y_{1}\right)\right) \wedge\left(g\left(T_{A}^{i}\right)\left(y_{2}\right), g\left(I_{A}^{i}\right)\left(y_{2}\right), g\left(F_{A}^{i}\right)\left(y_{2}\right)\right) \\
& =g(A)\left(y_{1}\right) \wedge g(A)\left(y_{2}\right) \text {. }
\end{aligned}
$$

Hence, the image of a neutrosophic multi subgroup is also a neutrosophic multi subgroup.

Theorem 5. Let $X_{1}, X_{2}$ be the classical groups and $g: X_{1} \rightarrow X_{2}$ be a group homomorphism. If $B$ is a neutrosophic multi subgroup of $X_{2}$, then the preimage $g^{-1}(B)$ is a neutrosophic multi subgroup of $X_{1}$. 
Proof. Let $B \in N M S\left(X_{2}\right)$ and $x_{1}, x_{2} \in X_{1}$. Since $g$ is a group homomorphism, the following inequality is obtained:

$$
\begin{aligned}
g^{-1}(B)\left(x_{1} x_{2}^{-1}\right) & \left(T^{i}{ }_{B}\left(g\left(x_{1} x_{2}^{-1}\right)\right), I_{B}^{i}\left(g\left(x_{1} x_{2}^{-1}\right)\right), F_{B}^{i}\left(g\left(x_{1} x_{2}^{-1}\right)\right)\right) \\
& =\left(T^{i}{ }_{B}\left(g\left(x_{1}\right) g\left(x_{2}\right)^{-1}\right), I^{i}{ }_{B}\left(g\left(x_{1}\right) g\left(x_{2}\right)^{-1}\right), F^{i} B\left(g\left(x_{1}\right) g\left(x_{2}\right)^{-1}\right)\right) \\
& \geq\left(T^{i} B\left(g\left(x_{1}\right)\right) \wedge T^{i}{ }_{B}\left(g\left(x_{2}\right)\right), I_{B}^{i}\left(g\left(x_{1}\right)\right) \vee I_{B}^{i}\left(g\left(x_{2}\right)\right), F_{B}^{i}\left(g\left(x_{1}\right)\right) \vee F^{i}\left(g\left(x_{2}\right)\right)\right) \\
& =\left(T^{i}{ }_{B}\left(g\left(x_{1}\right)\right), I_{B}^{i}\left(g\left(x_{1}\right)\right), F^{i}{ }_{B}\left(g\left(x_{1}\right)\right)\right) \wedge\left(T^{i}{ }_{B}\left(g\left(x_{2}\right)\right), I_{B}^{i}\left(g\left(x_{2}\right)\right), F_{B}^{i}\left(g\left(x_{2}\right)\right)\right) \\
& =g^{-1}(B)\left(x_{1}\right) \wedge g^{-1}(B)\left(x_{2}\right) .
\end{aligned}
$$

Therefore, $g^{-1}(B) \in N M S\left(X_{1}\right)$.

Definition 17. Let $X$ be a classical group. $A \in N M G(X)$; then, the compound function of $A$ and $A$ is defined as

$$
A \tilde{\circ} A(z)=\left\{z, T_{A \tilde{}}^{i} A(z), I_{A \tilde{}}^{i} A(z), F_{A \tilde{}}^{i} A(z): \forall z \in X\right\},
$$

where $T_{A \tilde{}}^{i}(z)=\left(\vee_{x y=z} T_{A}^{i}(y) \wedge T_{A}^{i}\left(z y^{-1}\right)\right), I_{A \tilde{}}^{i} A(z)=\left(\wedge_{x y=z} I_{A}^{i}(y) \vee I_{A}^{i}\left(z y^{-1}\right)\right)$ and $F_{A \check{ }}^{i}(z)=$ $\left(\wedge_{x y=z} F_{A}^{i}(y) \vee F_{A}^{i}\left(z y^{-1}\right)\right)$.

Theorem 6. Let $A \in N M S(X)$. Then, $A \in N M G(X)$ iff $A \tilde{\circ} A \tilde{\subseteq} A$ and $A \tilde{\subseteq} A^{-1}$.

Proof. Let $A \in N M S(X)$ and $x, y, z \in X$.

$$
\begin{aligned}
& \Rightarrow T^{i}{ }_{A}(x y) \geq T^{i}{ }_{A}(x) \wedge T^{i}{ }_{A}(y) \\
& \Rightarrow T^{i}{ }_{A}(z) \geq \vee\left\{T^{i}{ }_{A}(x) \wedge T^{i}{ }_{A}(y) ; x y=z\right\} \\
& =T^{i}{ }_{A \tilde{}}(z) \\
& \Rightarrow I^{i}{ }_{A}(x y) \leq T^{i}{ }_{A}(x) \vee I^{i}{ }_{A}(y) \\
& \Rightarrow I^{i}(z) \leq \wedge\left\{I^{i}{ }_{A}(x) \vee I^{i}{ }_{A}(y) ; x y=z\right\} \\
& =I^{i}{ }_{A}(z) \\
& \Rightarrow F^{i}{ }_{A}(x y) \leq F^{i}{ }_{A}(x) \vee T^{i}{ }_{A}(y) \\
& \Rightarrow F^{i}{ }_{A}(z) \leq \wedge\left\{F^{i}{ }_{A}(x) \vee F^{i}{ }_{A}(y) ; x y=z\right\} \\
& =F^{i}{ }_{A}(z)
\end{aligned}
$$

$\Rightarrow A \tilde{\circ} A \tilde{\subseteq} A$.

Now, by Proposition 2, we get the conditions. Conversely, suppose $A \tilde{\simeq} A \tilde{\subseteq} A$ and $A \subseteq \tilde{\subseteq} A^{-1}$

$$
\begin{aligned}
& \Rightarrow T_{A}^{i-1}(x) \geq T^{i}{ }_{A}(x) \text { but } T_{A}^{i-1}(x)=T_{A}^{i}\left(x^{-1}\right) \Rightarrow T^{i}{ }_{A}\left(x^{-1}\right) \geq T^{i}{ }_{A}(x) \\
& \Rightarrow I_{A}^{i}(x) \leq T_{A}^{i}(x) \text { but } I_{A}^{i-1}(x)=I_{A}^{i}{ }_{A}\left(x^{-1}\right) \Rightarrow I^{i}{ }_{A}\left(x^{-1}\right) \leq I^{i}{ }_{A}(x) \\
& \Rightarrow F_{A}^{i-1}(x) \leq F^{i}{ }_{A}(x) \text { but } F_{A}^{i-1}(x)=F_{A}^{i}{ }_{A}\left(x^{-1}\right) \Rightarrow F^{i}{ }_{A}\left(x^{-1}\right) \leq F_{A}^{i}(x)
\end{aligned}
$$

since $A \in N M S(X)$; then, to prove $A \in N M G(X)$, it enough to prove that $T_{A}^{i}\left(x y^{-1}\right) \geq T^{i}{ }_{A}(x) \wedge$ $T^{i}{ }_{A}(y), I_{A}^{i}\left(x y^{-1}\right) \leq I_{A}^{i}(x) \vee I_{A}^{i}(y)$ and $F_{A}^{i}\left(x y^{-1}\right) \leq F^{i}{ }_{A}(x) \vee F^{i}{ }_{A}(y) \forall x, y \in X$.

Now,

$$
\begin{aligned}
T_{A}^{i}\left(x y^{-1}\right) & \geq T^{i}{ }_{A \tilde{}}\left(x y^{-1}\right) \\
& =\vee_{z \in X}\left\{T^{i}{ }_{A}(z) \wedge T^{i}{ }_{A}\left(z^{-1} x y^{-1}\right)\right\} \\
& \geq\left\{T^{i}{ }_{A}(x) \wedge T^{i}{ }_{A}\left(y^{-1}\right) ; z=x\right\} \\
& \geq T^{i}{ }_{A}(x) \wedge T^{i}{ }_{A}(y) \\
I^{i}{ }_{A}\left(x y^{-1}\right) & \leq I^{i}{ }_{A \tilde{}}\left(x y^{-1}\right) \\
& =\wedge_{z \in X}\left\{I^{i}{ }_{A}(z) \vee I^{i}{ }_{A}\left(z^{-1} x y^{-1}\right)\right\} \\
& \leq\left\{I^{i}{ }_{A}(x) \vee I^{i}{ }_{A}\left(y^{-1}\right) ; z=x\right\} \\
& \leq I^{i}{ }_{A}(x) \vee I^{i}{ }_{A}(y)
\end{aligned}
$$




$$
\begin{aligned}
F^{i}{ }_{A}\left(x y^{-1}\right) & \leq F^{i}{ }_{A \tilde{}} A\left(x y^{-1}\right) \\
& =\wedge_{z \in X}\left\{F^{i}{ }_{A}(z) \vee F^{i}{ }_{A}\left(z^{-1} x y^{-1}\right)\right\} \\
& \leq\left\{F^{i}(x) \vee F^{i}{ }_{A}\left(y^{-1}\right) ; z=x\right\} \\
& \leq F^{i}{ }_{A}(x) \vee F^{i}{ }_{A}(y),
\end{aligned}
$$

hence the proof.

Corollary 3. Let $A \in N M S(X)$. Then, $A \in N M G(X)$ iff $A \tilde{o} A=A$ and $A \tilde{\subseteq} A^{-1}$.

Proof. Let $A \in N M G(X)$. Then,

$$
\begin{aligned}
T^{i}{ }_{A \tilde{}}(x) & =\vee\left\{T^{i}{ }_{A}(y) \wedge T^{i}{ }_{A}(z) ; y, z \in X \text { and } y z=x\right\} \\
& \geq\left\{T^{i}(e) \wedge T^{i}{ }_{A}\left(e^{-1} x\right)\right\} \\
& =T^{i}{ }_{A}(x) \\
I^{i}{ }_{A \tilde{}} A(x) & =\wedge\left\{I^{i}{ }_{A}(y) \vee I^{i}{ }_{A}(z) ; y, z \in X \text { and } y z=x\right\} \\
& \leq\left\{I^{i}(e) \vee I^{i}{ }_{A}\left(e^{-1} x\right)\right\} \\
& =I^{i}{ }_{A}(x) \\
F^{i}{ }_{A \tilde{}}(x) & =\wedge\left\{F^{i}{ }_{A}(y) \vee F^{i}{ }_{A}(z) ; y, z \in X \text { and } y z=x\right\} \\
& \leq\left\{F^{i}{ }_{A}(e) \vee F^{i}{ }_{A}\left(e^{-1} x\right)\right\} \\
& =F^{i}{ }_{A}(x) .
\end{aligned}
$$

Therefore, $A \tilde{\subseteq} A \tilde{\circ} A$.

Hence, by the above theorem, the proof is complete.

Theorem 7. Let $X$ be a classical group and $A, B \in N M S(X)$. If $A, B \in N M G(X)$, then $A \tilde{\cap} B \in N M G(X)$.

Proof. Let $x, y \in X$ be arbitrary:

$$
\begin{gathered}
\Rightarrow T_{A}^{i}\left(x y^{-1}\right) \geq T_{A}^{i}(x) \wedge T_{A}^{i}\left(y^{-1}\right), T_{B}^{i}\left(x y^{-1}\right) \geq T_{B}^{i}(x) \wedge T_{B}^{i}\left(y^{-1}\right) \\
I_{A}^{i}\left(x y^{-1}\right) \leq I_{A}^{i}(x) \vee I^{i}{ }_{A}\left(y^{-1}\right), I_{B}^{i}\left(x y^{-1}\right) \leq I_{B}^{i}(x) \vee T^{i}{ }_{B}\left(y^{-1}\right) \\
F_{A}^{i}\left(x y^{-1}\right) \leq F_{A}^{i}(x) \vee F^{i}{ }_{A}\left(y^{-1}\right), F_{B}^{i}\left(x y^{-1}\right) \leq F_{B}^{i}(x) \vee F_{B}^{i}\left(y^{-1}\right) .
\end{gathered}
$$

Now,

$$
\begin{aligned}
& T_{A \tilde{B} B}^{i}\left(x y^{-1}\right)=T_{A \tilde{\cap} B}^{i}(x) \wedge T_{A \tilde{B} B}^{i}\left(y^{-1}\right) \text { by definition intersection } \\
& \geq\left[T^{i}{ }_{A}(x) \wedge T^{i}{ }_{A}\left(y^{-1}\right)\right] \wedge\left[T^{i}{ }_{B}(x) \wedge T^{i}{ }_{B}\left(y^{-1}\right)\right] \\
& =\left[T^{i}{ }_{A}(x) \wedge T^{i}{ }_{B}(x)\right] \wedge\left[T^{i}{ }_{A}\left(y^{-1}\right) \wedge T_{B}^{i}\left(y^{-1}\right)\right] \text { by commutative property of minimum } \\
& \geq\left[T^{i}{ }_{A}(x) \wedge T^{i}{ }_{B}(x)\right] \wedge\left[T^{i}{ }_{A}(y) \wedge T_{B}^{i}(y)\right] \text { since } A, B \in N M G(X) \\
& =T_{A \cap \tilde{B}}^{i}(x) \wedge T_{A \tilde{}{ }^{i}}(y) \text { by definition intersection } \\
& \Rightarrow T_{A \tilde{n} B}^{i}\left(x y^{-1}\right) \geq T_{A \tilde{B} B}^{i}(x) \wedge T_{A \tilde{n} B}^{i}(y) \text { (1) } \\
& I_{A \tilde{n} B}^{i}\left(x y^{-1}\right)=I_{A \tilde{n} B}^{i}(x) \vee I_{A \tilde{n} B}^{i}\left(y^{-1}\right) \text { by definition intersection } \\
& \leq\left[I_{A}^{i}(x) \vee I^{i}{ }_{A}\left(y^{-1}\right)\right] \vee\left[I_{B}^{i}(x) \vee I_{B}^{i}{ }_{B}\left(y^{-1}\right)\right] \\
& =\left[I_{A}^{i}(x) \vee I^{i}{ }_{B}(x)\right] \vee\left[I_{A}^{i}\left(y^{-1}\right) \vee I^{i}{ }_{B}\left(y^{-1}\right)\right] \text { by commutative property of maximum } \\
& \leq\left[I_{A}^{i}(x) \vee I_{B}^{i}(x)\right] \vee\left[I^{i}{ }_{A}(y) \vee I_{B}{ }_{B}(y)\right] \text { since } A, B \in N M G(X) \\
& =I_{A \tilde{}}^{i} B(x) \vee I_{A \tilde{ก} B}^{i}(y) \text { by definition intersection } \\
& \Rightarrow I_{A \tilde{\cap} B}^{i}\left(x y^{-1}\right) \leq I_{A \tilde{\cap} B}^{i}(x) \wedge I_{A \tilde{B} B}^{i}(y)(2)
\end{aligned}
$$




$$
\begin{aligned}
& F_{A \tilde{\cap} B}^{i}\left(x y^{-1}\right)=F_{A \tilde{B} B}^{i}(x) \vee F_{A \tilde{B} B}^{i}\left(y^{-1}\right) \text { by definition intersection } \\
& \leq\left[F^{i}{ }_{A}(x) \vee F^{i}{ }_{A}\left(y^{-1}\right)\right] \vee\left[F_{B}^{i}(x) \vee F_{B}^{i}{ }_{B}\left(y^{-1}\right)\right] \\
& =\left[F^{i}{ }_{A}(x) \vee F^{i}{ }_{B}(x)\right] \vee\left[F^{i}{ }_{A}\left(y^{-1}\right) \vee F_{B}^{i}\left(y^{-1}\right)\right] \text { by commutative property of maximum } \\
& \leq\left[F_{A}^{i}(x) \vee F_{B}^{i}(x)\right] \vee\left[F^{i}{ }_{A}(y) \vee F_{B}^{i}(y)\right] \text { since } A, B \in N M G(X) \\
& =F_{A \tilde{ก} B}^{i}(x) \vee F_{A \tilde{ก} B}^{i}(y) \text { by definition intersection } \\
& \Rightarrow F_{A \tilde{n} B}^{i}\left(x y^{-1}\right) \leq F_{A \tilde{n} B}^{i}(x) \wedge F_{A \tilde{ก} B}^{i}(y)(3)
\end{aligned}
$$

From (1), (2) and (3), $A \tilde{\cap} B \in N M G(X)$, hence the proof.

Remark 1. Let $X$ be a classical group and $\left\{A_{i} ; i \in I\right\}$ be neutrosophic multiset on $X$. If $\left\{A_{i} ; i \in I\right\}$ is a family of $N M G(X)$ over $X$, then their intersection $\tilde{\bigcap}_{i \in I} A_{i}$ is also a $N M G(X)$ over $X$.

Proposition 6. Let $A, B \in N M G(X)$. Then, $T_{A \cup B}^{i}(x) \leq T_{A \cup B}^{i}\left(x^{-1}\right), I_{A \cup B}^{i}(x) \geq$ $I_{A \cup B}^{i}\left(x^{-1}\right), F_{A \cup B}^{i}(x) \geq F_{A \cup B}^{i}\left(x^{-1}\right)$.

Proof. Let $x, y \in X$. Now,

$$
\begin{aligned}
& T^{i}{ }_{A \cup B}\left(x^{-1}\right)=\vee\left\{T_{A}^{i}\left(x^{-1}\right), T_{B}^{i}\left(x^{-1}\right)\right\} \\
& \geq \vee\left\{T^{i}{ }_{A}(x), T_{B}^{i}(x)\right\} \text { since } A, B \in N M G(X) \\
& =T_{A \tilde{u} B}^{i}(x) \\
& I_{A \cup B}^{i}\left(x^{-1}\right)=\wedge\left\{I^{i}{ }_{A}\left(x^{-1}\right), I_{B}^{i}\left(x^{-1}\right)\right\} \\
& \leq \wedge\left\{I^{i}{ }_{A}(x), I_{B}{ }_{B}(x)\right\} \text { since } A, B \in N M G(X) \\
& =I_{A \tilde{}}^{i} B(x) \\
& F_{A \tilde{\cup} B}^{i}\left(x^{-1}\right)=\wedge\left\{F_{A}^{i}\left(x^{-1}\right), F_{B}^{i}\left(x^{-1}\right)\right\} \\
& \leq \wedge\left\{F^{i}{ }_{A}(x), F_{B}^{i}(x)\right\} \text { since } A, B \in N M G(X) \\
& =F_{A \cup B}^{i}(x) \text {, }
\end{aligned}
$$

hence the proof.

From this, it is clear that, if $A, B \in N M G(X)$, then $A \tilde{\cup} B \in N M G(X)$ iff $T_{A \cup B}^{i}(x y) \geq T_{A \sim B}^{i}(x) \wedge$ $T_{A \cup B}^{i}(y), I_{A \cup B}^{i}(x y) \leq T_{A \cup B}^{i}(x) \vee I_{A \cup B}^{i}(y), F_{A \cup B}^{i}(x y) \leq F_{A \cup B}^{i}(x) \vee F_{A \cup B}^{i}(y)$.

Corollary 4. Let $A, B \in N M G(X)$. Then, $A \tilde{\cup} B$ need not be an element of $N M G(X)$.

Example 7. Assume that $X=\{1,-1, i,-i\}$ is a classical group. Then,

$$
\begin{aligned}
A & =\{\langle 1 ;(0.5,0.3,0.2,0.1),(0.1,0.1,0.2,0.3),(0.2,0.3,0.4,0.5)\rangle, \\
& \langle-1 ;(0.7,0.6,0.4,0.3),(0.1,0.2,0.2,0.4),(0.2,0.5,0.4,0.3)\rangle\}, \\
& \langle i ;(0.6,0.4,0.3,0.2),(0.1,0.3,0.3,0.4),(0.1,0.2,0.4,0.6)\rangle, \\
& \langle-i ;(0.6,0.4,0.3,0.2),(0.1,0.3,0.3,0.4),(0.1,0.2,0.4,0.6)\rangle\}, \\
B \quad & =\{\langle 1 ;(0.5,0.6,0.6,0.4),(0.1,0.2,0.2,0.3),(0.2,0.4,0.4,0.5)\rangle, \\
& \langle-1 ;(0.7,0.6,0.4,0.3),(0.2,0.1,0.2,0.3),(0.3,0.4,0.5,0.3)\rangle\} \\
& \text { are NM-groups. } \\
A \cup B \quad & =\{\langle 1 ;(0.5,0.6,0.6,0.4),(0.1,0.1,0.2,0.3),(0.2,0.3,0.4,0.5)\rangle, \\
& \langle-1 ;(0.7,0.6,0.4,0.3),(0.1,0.2,0.2,0.3),(0.2,0.4,0.4,0.3)\rangle, \\
& \langle i ;(0.6,0.4,0.3,0.2),(0.1,0.3,0.3,0.4),(0.1,0.2,0.4,0.6)\rangle, \\
& \langle-i ;(0.6,0.4,0.3,0.2),(0.1,0.3,0.3,0.4),(0.1,0.2,0.4,0.6)\rangle\} .
\end{aligned}
$$

However, $T_{A \tilde{}}^{i} B(1) \geq T_{A \tilde{}}^{i}(i) \wedge T_{A \cup B}^{i}(-i)$ as $i .(-i)=1$. Then, $A \tilde{\cup} B \notin N M G(X)$.

Proposition 7. If $A \in N M G(X)$ and $X_{1}$ is a subgroup of $X$, then $A_{\left.\right|_{X_{1}}}$ (i.e., A restricted to $\left.X_{1}\right) \in N M-$ group $\left(X_{1}\right)$ and is a neutrosophic multi subgroup of $A$. 
Proof. Let $x, y \in X_{1}$. Then, $x y^{-1} \in X_{1}$. Now,

$$
\begin{aligned}
& T_{A_{\left.\right|_{1}}}^{i}\left(x y^{-1}\right)=T_{A}^{i}\left(x y^{-1}\right) \geq T_{A}^{i}(x) \wedge T_{A}^{i}(y)=T_{A_{\mid X_{1}}}^{i}(x) \wedge T_{A_{\mid X_{1}}}^{i}(y), \\
& I^{i}{ }_{A_{\mid X_{1}}}\left(x y^{-1}\right)=I^{i}{ }_{A}\left(x y^{-1}\right) \leq I^{i}{ }_{A}(x) \vee I^{i}{ }_{A}(y)=I^{i}{ }_{A_{\mid X_{1}}}(x) \vee I^{i}{ }_{A_{\mid X_{1}}}(y), \\
& F_{A_{\left.\right|_{X_{1}}}}\left(x y^{-1}\right)=F_{A}^{i}\left(x y^{-1}\right) \leq F^{i}{ }_{A}(x) \vee F^{i}{ }_{A}(y)=F^{i}{ }_{A_{\left.\right|_{X_{1}}}}(x) \vee F^{i}{ }_{A_{\left.\right|_{X_{1}}}}(y) \text {. }
\end{aligned}
$$

The second part is trivial.

Definition 18. Let $A \in N M G(X)$ and $B \in N M G(Y)$ be two neutrosophic multi groups over the groups $X$ and $Y$, respectively. Then, the Cartesian product of $A$ and $B$ is defined as $(A \tilde{\times} B)(x, y)=A(x) \tilde{\times} B(y)$ where

$$
A \tilde{\times} B=\left\{(x, y), T_{A \tilde{\times} B}^{i}(x, y), I_{A \tilde{\times} B}^{i}(x, y), F_{A \tilde{\times} B}^{i}(x, y):(x, y) \in X \times Y\right\},
$$

where $T_{A \tilde{\times} B}^{i}(x, y)=T^{i}{ }_{A}(x) \vee T_{B}^{i}(y), I_{A \tilde{\times} B}^{i}(x, y)=I_{A}^{i}(x) \wedge I_{B}^{i}(y), F_{A \tilde{\times} B}^{i}(x, y)=F^{i}{ }_{A}(x) \wedge F_{B}^{i}(y)$.

Example 8. Assume that $\left(Z_{2},+\right)$ and $\left(Z_{3},+\right)$ are classiccal groups. Define the neutrosophic multi group $A$ on $\left(Z_{2},+\right)$ and $B$ on $\left(Z_{3},+\right)$ as follows:

$$
\begin{aligned}
A & =\{\langle 0 ;(0.6,0.5,0.4,0.2),(0.2,0.2,0.3,0.4),(0.2,0.3,0.4,0.6)\rangle, \\
& \langle 1 ;(0.6,0.5,0.4,0.3),(0.2,0.1,0.2,0.3),(0.1,0.2,0.3,0.4)\rangle\} \\
B & =\{\langle 0 ;(0.7,0.6,0.5,0.4),(0.2,0.2,0.3,0.4),(0.3,0.4,0.5,0.5)\rangle, \\
& \langle 1 ;(0.7,0.6,0.5,0.3),(0.3,0.4,0.3,0.4),(0.3,0.2,0.5,0.5)\rangle, \\
& \langle 2 ;(0.8,0.7,0.5,0.4),(0.1,0.3,0.2,0.3),(0.2,0.1,0.3,0.5)\rangle\} . \\
A \tilde{\times} B \quad & =\{\langle(0,0) ;(0.6,0.5,0.4,0.2),(0.2,0.2,0.3,0.4),(0.2,0.3,0.4,0.6)\rangle, \\
& \langle(0,1) ;(0.6,0.5,0.4,0.2),(0.3,0.4,0.3,0.4),(0.3,0.3,0.5,0.6)\rangle, \\
& \langle(0,2) ;(0.6,0.5,0.4,0.2),(0.2,0.3,0.3,0.4),(0.2,0.3,0.4,0.6)\rangle, \\
& \langle(1,0) ;(0.6,0.5,0.4,0.3),(0.2,0.2,0.3,0.4),(0.3,0.4,0.5,0.5)\rangle, \\
& \langle(1,1) ;(0.6,0.5,0.4,0.3),(0.3,0.4,0.3,0.4),(0.3,0.2,0.5,0.5)\rangle, \\
& \langle(1,2) ;(0.6,0.5,0.4,0.3),(0.2,0.3,0.2,0.3),(0.2,0.2,0.3,0.5)\rangle\} .
\end{aligned}
$$

Then, $A \tilde{\times} B$ is a neutrosophic multi group.

Theorem 8. Let $A, B \in N M G(X)$. The cartesian product of $A$ and $B$ is denoted by $A \tilde{\times} B \in N M G(X)$.

Proof. From the Theorem 1, it is clear that a $N M G(X)$ is a neutrosophic multi group:

$$
\begin{aligned}
& T_{A \tilde{\times} B}^{i}\left(\left(x_{1}, y_{1}\right),\left(x_{2}, y_{2}\right)^{-1}\right)=T_{A \tilde{\times} B}^{i}\left(x_{1} x_{2}^{-1}, y_{1} y_{2}^{-1}\right) \\
& =T^{i}{ }_{A}\left(x_{1} x_{2}^{-1}\right) \wedge T_{B}^{i}\left(y_{1} y_{2}^{-1}\right) \\
& \geq\left(T^{i}{ }_{A}\left(x_{1}\right) \wedge T^{i}{ }_{A}\left(x_{2}\right)\right) \wedge\left(T_{B}^{i}\left(y_{1}\right) \wedge T^{i}{ }_{B}\left(y_{2}\right)\right) \\
& =\left(T^{i}{ }_{A}\left(x_{1}\right) \wedge T_{B}^{i}\left(y_{1}\right)\right) \wedge\left(T^{i}{ }_{A}\left(x_{2}\right) \wedge T^{i}{ }_{B}\left(y_{2}\right)\right) \\
& =T_{A \tilde{\times} B}^{i}\left(x_{1}, y_{1}\right) \wedge T_{A \tilde{x} B}^{i}\left(x_{2}, y_{2}\right) \\
& I_{A \tilde{\times} B}^{i}\left(\left(x_{1}, y_{1}\right),\left(x_{2}, y_{2}\right)^{-1}\right)=I_{A \tilde{\times} B}^{i}\left(x_{1} x_{2}^{-1}, y_{1} y_{2}^{-1}\right) \\
& =I_{A}^{i}\left(x_{1} x_{2}^{-1}\right) \vee I_{B}^{i}\left(y_{1} y_{2}^{-1}\right) \\
& \leq\left(I^{i}{ }_{A}\left(x_{1}\right) \vee I^{i}{ }_{A}\left(x_{2}\right)\right) \vee\left(I_{B}{ }_{B}\left(y_{1}\right) \vee I^{i}{ }_{B}\left(y_{2}\right)\right) \\
& =\left(I^{i}{ }_{A}\left(x_{1}\right) \vee I_{B}^{i}\left(y_{1}\right)\right) \vee\left(I^{i}{ }_{A}\left(x_{2}\right) \vee I_{B}^{i}\left(y_{2}\right)\right) \\
& =I_{A \tilde{\times} B}^{i}\left(x_{1}, y_{1}\right) \vee I_{A \tilde{\times} B}^{i}\left(x_{2}, y_{2}\right)
\end{aligned}
$$


and

$$
\begin{aligned}
F_{A \tilde{\times} B}^{i}\left(\left(x_{1}, y_{1}\right),\left(x_{2}, y_{2}\right)^{-1}\right) & =F_{A \tilde{\times} B}^{i}\left(x_{1} x_{2}^{-1}, y_{1} y_{2}^{-1}\right) \\
& =F^{i}{ }_{A}\left(x_{1} x_{2}^{-1}\right) \vee F^{i}{ }_{B}\left(y_{1} y_{2}^{-1}\right) \\
& \leq\left(F_{A}^{i}\left(x_{1}\right) \vee F^{i}{ }_{A}\left(x_{2}\right)\right) \vee\left(F_{B}^{i}\left(y_{1}\right) \vee F_{B}^{i}\left(y_{2}\right)\right) \\
& =\left(F^{i}{ }_{A}\left(x_{1}\right) \vee F_{B}^{i}\left(y_{1}\right)\right) \vee\left(F^{i}{ }_{A}\left(x_{2}\right) \vee F_{B}^{i}\left(y_{2}\right)\right) \\
& =F^{i}{ }_{A \tilde{\times} B}\left(x_{1}, y_{1}\right) \vee F_{A \tilde{x} B}^{i}\left(x_{2}, y_{2}\right)
\end{aligned}
$$

for all $x, y \in X$ and $i=1,2, \ldots, P$-hence the proof.

\section{Conclusions}

The concept of a group is of fundamental importance in the study of algebra. In this paper, the algebraic structure of neutrosophic multiset is introduced as a neutrosophic multigroup. The neutrosophic multigroup is a generalized case of intuitionistic fuzzy multigroup and fuzzy multigroup. The various basic operations, definitions and theorems related to neutrosophic multigroup have been discussed. The foundations which we made through this paper can be used to get an insight into the higher order structures of group theory.

Author Contributions: All authors contributed equally.

Funding: This research received no external funding.

Conflicts of Interest: The authors declare no conflict of interest.

\section{Abbreviations}

The following abbreviations are used in this manuscript:

NMG Neutrosophic Multigroup

NMS Neutrosophic Multiset

IFMS Intuitionistic Fuzzy Multiset

\section{References}

1. Zadeh, L.A. Fuzzy Sets. Inf. Control 1965, 8, 338-353. [CrossRef]

2. Sebastian, S.; Ramakrishnan, T.T. Multi-Fuzzy Sets. Int. Math. Forum 2010, 5, 2471-2476.

3. Atanassov, K. Intuitionistic fuzzy sets. Fuzzy Set Syst. 1986, 20, 87-96. [CrossRef]

4. Shinoj, T.K.; John, S.S. Intuitionistic fuzzy multisets and its application in medical diagnosis. World Acad. Sci. Eng. Technol. 2012, 6, 1418-1421.

5. Abdullah, S.; Naeem, M. A new type of interval valued fuzzy normal subgroups of groups. New Trends Math. Sci. 2015, 3, 62-77.

6. Mordeson, J.N.; Bhutani, K.R.; Rosenfeld, A. Fuzzy Group Theory; Springer: Berlin/Heidelberg, Germany, 2005.

7. Liu, Y.L. Quotient groups induced by fuzzy subgroups. Quasigroups Related Syst. 2004, 11, 71-78.

8. Baby, A.; Shinoj, T.K.; John, S.J. On Abelian Fuzzy Multi Groups and Orders of Fuzzy Multi Groups. J. New Theory 2015, 5, 80-93.

9. Muthuraj, R.; Balamurugan, S. Multi-Fuzzy Group and its Level Subgroups. Gen 2013, 17, 74-81.

10. Tella, Y. On Algebraic Properties of Fuzzy Membership Sequenced Multisets. Br. J. Math. Comput. Sci. 2015, 6, 146-164. [CrossRef]

11. Fathi, M.; Salleh, A.R. Intuitionistic fuzzy groups. Asian J. Algebra 2009, 2, 1-10. [CrossRef]

12. Li, X.P.; Wang, G.J. (1, a)-Homomorphisms of Intuitionistic Fuzzy Groups. Hacettepe J. Math. Stat. 2011, 40, 663-672.

13. Palaniappan, N.; Naganathan, S.; Arjunan, K. A study on Intuitionistic L-fuzzy Subgroups. Appl. Math. Sci. 2009, 3, 2619-2624.

14. Sharma, P.K. Homomorphism of Intuitionistic fuzzy groups. Int. Math. Forum 2011, 6, 3169-3178.

15. Sharma, P.K. On the direct product of Intuitionistic fuzzy subgroups. Int. Math. Forum 2012, 7, 523-530.

16. Sharma, P.K. On intuitionistic fuzzy abelian subgroups. Adv. Fuzzy Sets Syst. 2012, 12, 1-16. 
17. Xu, C.Y. Homomorphism of Intuitionistic Fuzzy Groups. In Proceedings of the 2007 International Conference on Machine Learning and Cybernetics, Hong Kong, China, 19-22 August 2007.

18. $\mathrm{Li}, \mathrm{X}$. Homomorphism and isomorphism of the intuitionistic fuzzy normal subgroups. BUSEFAL 2001, 85, 162-175.

19. Yuan, X.H.; Li, H.X.; Lee, E.S. On the definition of the intuitionistic fuzzy subgroups. Comput. Math. Appl. 2010, 59, 3117-3129. [CrossRef]

20. Shinoj, T.K.; John, S.J. Intuitionistic fuzzy multigroups. Ann. Pure Appl. Math. 2015, 9, 131-143.

21. Wang, H.; Smarandache, F.Y.; Zhang, Q.; Sunderraman, R. Single valued neutrosophic sets. Multispace Multistruct. 2010, 4, 410-413.

22. Smarandache, F. A Unifying Field in Logics; Infinite Study: Conshohocken, PA, USA, 1998.

23. Agboola, A.; Akwu, A.D.; Oyebo, Y.T. Neutrosophic groups and subgroups. Math. Comb. 2012, 3, 1-9.

24. Ali, M.; Smarandache, F. Neutrosophic Soluble Groups, Neutrosophic Nilpotent Groups and Their Properties. In Proceedings of the Annual Symposium of the Institute of Solid Mechanics (SISOM 2015), Bucharest, Romania, 21-22 May 2015.

25. Cetkin, V.; Aygün, H. An approach to neutrosophic subgroup and its fundamental properties. J. Intell. Fuzzy Syst. 2015, 29.5, 1941-1947. [CrossRef]

26. Shabir, M.; Ali, M.; Naz, M.; Smarandache, F. Soft neutrosophic group. Neutrosophic Sets Syst. 2013, 1, 13-25.

27. Deli, I.; Broumi, S.; Smarandache, F. On neutrosophic multisets and its application in medical diagnosis. J. New Theory 2015, 6, 88-98.

28. Ye, S.; Ye, J. Dice Similarity Measure between Single Valued Neutrosophic Multisets and Its Application in Medical Diagnosis. Neutrosophic Sets Syst. 2014, 6, 49-54.

29. Ye, S.; Fu, J.; Ye, J. Medical Diagnosis Using Distance-Based Similarity Measures of Single Valued Neutrosophic Multisets. Neutrosophic Sets Syst. 2015, 7, 47-52.

30. Rosenfeld, A. Fuzzy groups. J. Math. Anal. Appl. 1971, 35, 512-517. [CrossRef]

31. Şahin, M.; Uluçay, V.; Olgun, N.; Kilicman, A. On neutrosophic soft lattices. Afr. Matematika 2017, $28,379-388$.

32. Şahin, M.; Olgun, N.; Kargın, A.; Uluçay, V. Isomorphism theorems for soft G-modules. Afr. Matematika 2018, 29, 1-8. [CrossRef]

33. Uluçay, V.; Kiliç, A.; Yildiz, I.; Şahin, M. A new approach for multi-attribute decision-making problems in bipolar neutrosophic sets. Neutrosophic Sets Syst. 2018, 23, 142-159.

34. Şahin, M.; Deli, I.; Ulucay, V. Extension principle based on neutrosophic multi-sets and algebraic operations. J. Math. Ext. 2018, 12, 69-90.

35. Hu, Q.; Zhang, X. New Similarity Measures of Single-Valued Neutrosophic Multisets Based on the Decomposition Theorem and Its Application in Medical Diagnosis. Symmetry 2018 , 10, 466. [CrossRef]

36. Smarandache, F.; Şahin, M.; Kargın, A. Neutrosophic Triplet G-Module. Mathematics 2018, 6, 53. [CrossRef]

37. Şahin, M.; Olgun, N.; Uluçay, V. Normed Quotient Rings. New Trends Math. Sci. 2018, 6, 52-58.

38. Uluçay, V.; Şahin, M.; Olgun, N. Soft Normed Rings. SpringerPlus 2016, 5, 1950. [CrossRef] [PubMed]

39. Sharma, P.K. Modal Operator F, B in Intuitionistic Fuzzy Groups. Ann. Pure Appl. Math. 2014, 7, 19-28.

(C) 2019 by the authors. Licensee MDPI, Basel, Switzerland. This article is an open access article distributed under the terms and conditions of the Creative Commons Attribution (CC BY) license (http:/ / creativecommons.org/licenses/by/4.0/). 